\title{
Internet of Vehicles: Sensing Aided Transportation Information Collection and Diffusion
}

\author{
Jingjing Wang, Student Member, IEEE, Chunxiao Jiang, Senior Member, IEEE, Zhu Han, Fellow, IEEE, \\ Yong Ren, Senior Member, IEEE and Lajos Hanzo Fellow, IEEE
}

\begin{abstract}
In view of the emergence and rapid development of the Internet of Vehicles (IoV) and cloud computing, intelligent transport systems (ITS) are beneficial in terms of enhancing the quality and interactivity of urban transportation services, of reducing costs and resource wastage, as well as of improving the traffic management capability. Efficient traffic management relies on the accurate and prompt acquisition as well as diffusion of traffic information. To achieve this, research is mostly focused on optimizing the mobility models and communication performance. However, considering the escalating scale of IoV networks, the interconnection of heterogeneous smart vehicles plays a critical role in enhancing the efficiency of traffic information collection and diffusion. In this paper, we commence by establishing a weighted and undirected graph model for IoV sensing networks and verify its time-invariant complex characteristics relying on a real-world taxi-GPS dataset. Moreover, we propose an IoV aided local traffic information collection architecture, a sink node selection scheme for the information influx as well as an optimal traffic information transmission model. Our simulation results and theoretical analysis show the efficiency and feasibility of our proposed models.
\end{abstract}

Index Terms-Internet of Vehicles (IoV); intelligent transport systems (ITS); traffic information collection; information diffusion.

\section{INTRODUCTION}

With the emergence of intelligent transport systems (ITS), travellers are better informed and use the ever-smarter transport networks more safely [1] [2]. The Internet of Vehicles (IoV) paradigm [3] [4] assists in connecting the vehicles to the Internet, which are well-endowed with communication units and new sensors, in order to support information-exchange, identification, localization, monitoring and managing [5]. During the last decades, the total number of vehicles in some parts of the world increased faster than the population [6]. Hence, relying on frequent information exchange as well as other interactions and advanced computing capabilities, the IoV aided

J. Wang and Y. Ren are with the Department of Electronic Engineering, Tsinghua University, Beijing, 100084, China. E-mail: chinaeephd@gmail.com, reny@tsinghua.edu.cn.

C. Jiang is with Tsinghua Space Center, Tsinghua University, Beijing, 100084, China. E-mail: jchx@tsinghua.edu.cn.

Z. Han is with the University of Houston, Houston, TX 77004 USA, and also with the Department of Computer Science and Engineering, Kyung Hee University, Seoul, South Korea. Email: zhan2@uh.edu.

L. Hanzo is with the School of Electronics and Computer Science, University of Southampton, Southampton, SO17 1BJ U.K. Email: lh@ecs.soton.ac.uk.

This research was supported by the NSFC China project 61371079. L. Hanzo would like to acknowledge the financial support of the European Research Council's Advanced Fellow Grant and of the Royal Society's Wolfson Research Merit Award.

(c)IEEE TVT 2018 vehicular network is beneficial both in terms of increasing the efficiency of traffic management, as well as providing compelling services for ITS, such as traffic management, smart warning, multimedia access, environmental monitoring, etc. Based on the maturing vehicular ad hoc networks (VANETs), IoV aided traffic information collection and diffusion may be invoked both for vehicle-to-vehicle (V2V) communications and vehicle-to-infrastructure (V2I) interactions, as well as for the sensing, transmission and integration of important information related to a city's traffic-flow for preventing traffic jams.

From a macroscopic perspective, the IoV aided ITS can be deemed to be a complex system [7] [8]. First of all, the IoV aided ITS is associated with a tremendous network size. Specifically, tens of thousands of vehicles are on the road every day, communicating with the road-side infrastructure, especially in metropoles, such as Beijing and New York City. Hence, we need the statistical analysis to characterize and model the behaviour of these smarts units. Secondly, the heterogeneous and hierarchical network structures and node types relying on the IoV technologies result in more complex interactions. Vehicular networks, the Internet, infrastructural networks, traditional terrestrial telecommunication networks and even the satellite networks are increasingly capable of communicating with each other anytime and anywhere, which requires that the ITS is suitably equipped for handling a diverse variety of communication entities. Finally, the IoV based ITS has a complex time-space structure. The mobility of the vehicles on the road leads to a dynamically evolving topology. Moreover, both their movement trajectory and position distribution are affected by the terrain and population distribution in the city.

Due to the cooperation among vehicles, diverse sources of information may be sensed, transmitted and integrated in the context of IoV aided ITS. However, in view of the aforementioned complex time-space characteristics, the IoV aided ITS has irregularly fluctuating dynamic topological structures, which gives rise to grave challenges. Although cellular communication and networking technologies support convenient communications as well as some entertainment services for drivers and passengers, they are not well-suited for some of the sensing aided traffic information collection and diffusion services in V2V or V2I communications [9] [10].

In the literature of the traffic information collection and diffusion, Palazzi et al. [11] proposed an inter-vehicular communication architecture capable of promptly propagating their messages over a vehicular network. Specifically, relying both 
on the features of device-to-device (D2D) and on vehicular networks, Cheng et al. [12] presented a reliable and efficient D2D-aided vehicular communications framework for ITS. Furthermore, relying on an ad hoc inter-vehicle network, a decentralized traffic information dissemination mechanism was conceived by Wischhof et al. [13]. By exploiting the moderately delay-tolerant nature of traffic message propagation, an analytical model based on a traditional bidirectional highway scene was presented by Agarwal et al. [14]. Furthermore, Chaqfeh et al. [15], surveyed the family of information broadcasting techniques relying both on data caching and on redundancy reduction. In [16], Panichpapiboon et al. reduced the delivery overhead by selecting only a subset of relay nodes for rebroadcasting information based on positioning information. Recently, Zhu et al. [17] proposed a mobile data offloading system that integrated the classic cellular network and opportunistic vehicular communications. In [18], Zhang et $a l$. proposed a rapid traffic information dissemination model for a large-scale urban road network, which had a degree of autonomy and a high traffic information dissemination efficiency. Moreover, Kim et al. [19] maximized the data dissemination success probability under the practical condition that the size of local data storage was limited and that the wireless connectivity table was unknown. This was achieved by a greedy online learning algorithm. Furthermore, a novel framework was proposed by Rémy et al. in [20] [21] for a centralized vehicular network organization based on a 4G Long Term Evolution (LTE) network. However, these contributions primarily focused their attention on the topology characteristics and on the communication performance of vehicular networks instead of considering the quantity, density and heterogeneity of smart nodes in an IoV sensing aided network [22] [23] [24] [25]. These challenges inspired us to conceive this article on the architecture and key technologies of IoV sensing aided traffic information collection and diffusion [26]. In this paper, considering the complex characteristics of IoV networks, relying on a real-world dataset, we studied the IoV aided traffic information collection and diffusion by providing the following original contributions.

- This is the first treatise establishing a weighted and undirected graph model of IoV sensing networks. Moreover, we define the communication impedance of both the nodes and of the links based on their communication performances and network parameters.

- We invoke complex network based techniques for analyzing the topology of the IoV network constructed by the taxi-GPS database of Beijing city [27]. Based on these parameters, we characterize the time-invariance of the IoV network and study the relationship between their topology and communication performance, which plays a vital role in urban traffic control and management.

- We propose an IoV aided local traffic information collection architecture, and an efficient sink node selection scheme for supporting information influx as well as an optimal traffic information transmission model relying on the defined communication impedance in order to improve the information transfer efficiency.

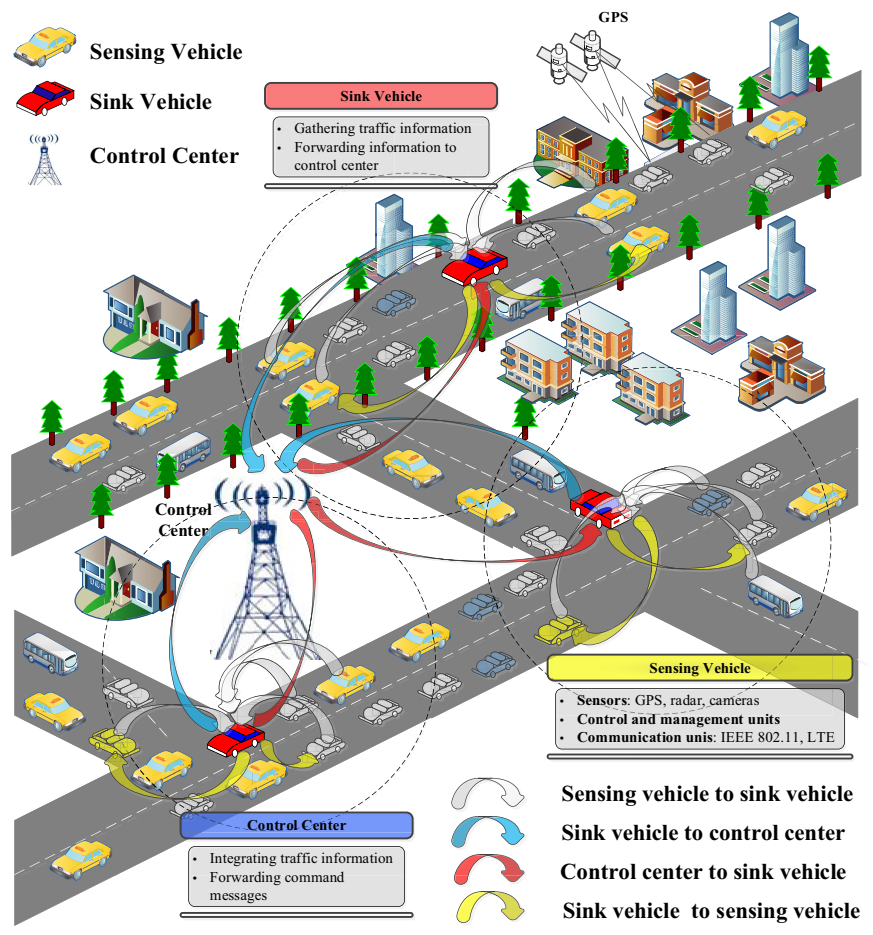

Fig. 1. The scenario of IoV aided traffic information collection and diffusion.

The remainder of the article is outlined as follows. In Section II, we present the system model of the IoV sensing aided traffic information collection and diffusion. Section III establishes a weighted and undirected graph model of the IoV network and identifies its key parameters as well as their characteristics. In Section IV, we propose an IoV aided traffic information collection architecture, a sink-node selection scheme for information influx as well as an information flow control algorithm. Section V characterizes the performance of the proposed algorithms, followed by our conclusions in Section VI.

\section{SYSTEM MODEL}

Traditional traffic management policies, such as odd-even day vehicle bans, license plate quota, etc., which may relieve the traffic congestions to some extent are unable to take into account the road-network's conditions, nor do they consider the urban population distribution and the peak/off-peak time factor. More efficient traffic management can be established by exploiting seamless information exchange and coordination across the entire IoV network. Specifically, with the aid of an IoV network, the traffic flow prediction mechanism is capable of relieving roads from heavy traffic and/or predicting the peak-traffic times and locations relying on historical observation data and real-time GPS location information. Moreover, traffic light scheduling management may be invoked to control traffic flow in order to approach the maximum network throughput. The vehicle speed management system broadcasts the notification of speed limits, which is intended to both ensure traffic safety and to beneficially exploit the road conditions. This is achieved by planning and recommending 
optimal driving routes for maintaining a smooth traffic flow, as well as providing constructive feedback for future roadnetwork design. In this section we propose an IoV assisted network architecture for improving the efficiency of both collecting and diffusing traffic information.

As for top-level urban traffic management, we need global information for making final decisions. As shown in Fig. 1, the vehicles should be equipped with sensors, control and management units, and communication units in order to fulfil certain tasks. In addition to the wide-spread classic sensors, such as GPS, radar, cameras, and so on, the vehicles may also be equipped with specific sensors depending on their missions. Moreover, the control and management units are responsible for the collaboration of each part. The communication units are composed of multiple modules obeying various protocols, such as IEEE 802.11, LTE, and so on, in order to support different communication purposes. The control center has a powerful data fusion, information processing and communication capability for integrating traffic information and for forwarding command messages. Since each vehicle has a limited sensing scope and computational capability, the information collected from each sensing vehicle has to be aggregated by sink vehicles (gateways), whose responsibility is to gather the information from the other nodes in the ITS and to forward the traffic information to the control center. Then, these sink vehicles can take charge of broadcasting the traffic control messages from the control center to the other vehicles.

The IoV-aided traffic management relies on three stages. First, we determine the IoV-aided sensing architecture, where the vehicles are classified into several subdomains in terms of their geographic location as well as their communication environment. The vehicles equipped with specific sensors collect the relevant traffic information within each subdomain. Secondly, in each subdomain, one of the sink vehicles (the red one in Fig. 1) is selected as the gateway, and the remaining sensing vehicles transmit their traffic information to the sink vehicle. Moreover, all the gateways forward the aggregated traffic information to the traffic control center. Thirdly, upon receiving the traffic control messages from the control center, the sink vehicles broadcast the relevant information to the remaining vehicles.

In the following, we first embark on modeling the IoV-aided vehicular network relying on complex network theory [7] [8], and then study the relevant techniques of the aforementioned three stages invoked for efficient traffic management.

\section{A Weighted And Undirected GRAPH Model FOR IOV NETWORKS}

Let us now construct a weighted and undirected graph model of the IoV-based traffic information collection and diffusion, where each interaction represents some traffic information transmission between a pair of vehicles. We rely on a specific weighting of the nodes for indicating the traffic information collection and distribution capability of each smart unit. Moreover, the weighting quantifies the performance of each information diffusion link, which are related to the fading, to the environmental impairments, to the cellular handover, etc. The following assumptions are stipulated to simplify our weighting process:

- All the vehicles or road-side infrastructure elements have an identical communication capability. A pair of nodes are only capable of communicating with each other, provided that they are within a specified maximum information transmission range $r$. Based on the idealized simplifying assumption of having instantaneous wireless information transmission, the main communication delay depends on the store-and-forward process of each node.

- Additive White Gaussian Noise (AWGN) is assumed, which is wide-sense stationary (WSS). Furthermore, a low-complexity empirical model is invoked for characterizing the urban wireless communication channel [28]. Additionally, we neglect the cellular coverage gaps.

Relying on the aforementioned assumptions, we propose the concept of node/link communication impedance quantifying the above-mentioned weighting, in order to characterize the node/link performance. Sommer et al. [28] proposed a computationally efficient empirical obstacle model for characterizing the radio propagation in urban environments, which considered the large-scale path loss, deterministic small-scale fading as well as the probabilistic attenuation effects. The total path loss is given by $L_{x}=L_{\text {freespace }}+L_{\text {obs }}$, where $L_{\text {freespace }}$ represents the best-case Line-of-Sight (LOS) propagation model between transmitters and receivers, and $L_{o b s}$ represents the additional attenuation imposed by obstacles, which are given by:

$$
L_{\text {freespace }}[\mathrm{dB}]=10 \lg \left(\frac{16 \pi^{2}}{\lambda^{2}} d^{\kappa}\right),
$$

as well as

$$
L_{o b s}[\mathrm{~dB}]=\beta_{1} n+\beta_{2} d_{m},
$$

where $\lambda$ is the wavelength and $d$ represents the distance between the source node and the destination node. Furthermore, $n$ denotes the number of occurrences that the obstacle is intersected by the LOS and $d_{m}$ is the total length of the obstacles' intersection. We assume that the path loss exponent is $\kappa=2.2$ and the associated calibration factors in Eq. (2) are $\beta_{1}=9 \mathrm{~dB}$ per wall and $\beta_{2}=0.4 \mathrm{~dB}$ per meter, respectively.

The reduction of the cellular radius is also beneficial in terms of increasing the system's achievable capacity. We assume that the cell is a circle with radius $r_{c}$ and we assume having no coverage dead zones. The number of handovers is denoted by $n_{i j}$.

When relying on the urban wireless channel model and ultra dense cellular scenarios resulting in numerous handovers, as well as on the concepts of node degrees and betweenness centralities $^{1}$ of the complex network [29], we define the weighting of the communication links connecting node $i$ and node $j$ under the condition of $d_{i j} \leq r$, which may be termed

\footnotetext{
${ }^{1}$ The betweenness centrality of a node is a measure of centrality in a graph based on shortest paths. It is defined by the number of shortest paths that pass through the node quantified in terms of the number of nodes rather than distance.
} 
as the link's communication impedance $R_{i j}$, as follows:

$R_{i j}=\alpha_{1}\left(k_{i} B_{i}+k_{j} B_{j}\right)^{\psi_{1}}+\alpha_{2} L_{i j}{ }^{\psi_{2}}-\alpha_{3}\left(E N R / d_{i j}\right)^{\psi_{3}}+\alpha_{4} n_{i j}$.

For $d_{i j}>r$, we have $R_{i j}=\infty$, while $k_{i}$ represents the node degree of vehicle $i$ and $B_{i}$ denotes its betweenness centrality. The energy per bit to noise power spectral density ratio is given by $E N R^{2}$ [30]. Furthermore, $\alpha_{1}, \alpha_{2}, \alpha_{3}$ and $\alpha_{4}$ are additional characteristic parameters, which vary as a function of the network's topology, and $\psi_{1}, \psi_{2}, \psi_{3}$ are nonlinear control parameters. $L_{i j}$ denotes the pass loss between vehicle $i$ and vehicle $j$. Physically, the communication impedance takes into account the node's between centrality, transmission distance, unit energy to noise ratio as well as the number of cellular handovers. First of all, a vehicle having a high node-degree and a high betweenness centrality plays a much more important role in the communication missions, since they potentially contribute to a long store-and-forward delay and to a high probability of blocking. Secondly, a high transmission distance between a pair of nodes results in a high path loss and a high power consumption but a potentially reduced delay, since less store-and-forward processes are involved. Furthermore, a small cellular radius leads to a high number of handovers $n_{i j}$, which further increases the transmission delay and degrades the communication performance attained. Finally, a high average ENR per unit distance contributes to high-quality communication, which corresponds to a low communication link impedance.

In a nutshell, we have constructed a weighted and undirected complex network graph model $G=(V, E, R)$ for the vehicular network considered in Fig. 2, where $V$ represents the set of smart units and $E$ denotes the set of graph-edges representing the interactions amongst the nodes. Moreover, the set of weights, namely the communication link impedances $R$, quantifies the traffic information diffusion performance. In the following, relying on the proposed graph model, we will focus our attention on the IoV aided traffic information collection and diffusion process.

\section{IOV AIDED TRAFFIC INFORMATION COLLECTION AND DIFFUSION}

\section{A. IoV aided Traffic Information Collection Architecture}

Having a well-designed IoV-aided sensing architecture critically hinges on the traffic information diffusion coverage range of Fig. 2. In [31], Yang et al. derived the achievable rate $\Lambda$ of the uplink transmission of user $k$, which is given by:

$$
\Lambda \triangleq(1-\tau-\varsigma) \mathrm{E}[\log (1+\gamma)],
$$

where $\gamma$ represents the signal-to-interference-plus-noise-ratio (SINR), which is characterized by the channel model parameters and antenna parameters. Furthermore, $\tau$ is the channel estimation duration and $\varsigma$ denotes the propagation delay, but we only consider the value of $\Lambda$, rather than its individual parameters. Hence, we define the node's communication impedance $R_{i}$ as follows:

$$
R_{i}=\xi_{1}\left(k_{i} B_{i}\right)^{\omega_{1}}+\xi_{2} \Lambda^{\omega_{2}}, i=1,2, \ldots, N
$$

${ }^{2}$ ENR is a normalized signal-to-noise ratio (SNR) measure, which is defined as the SNR per bit.

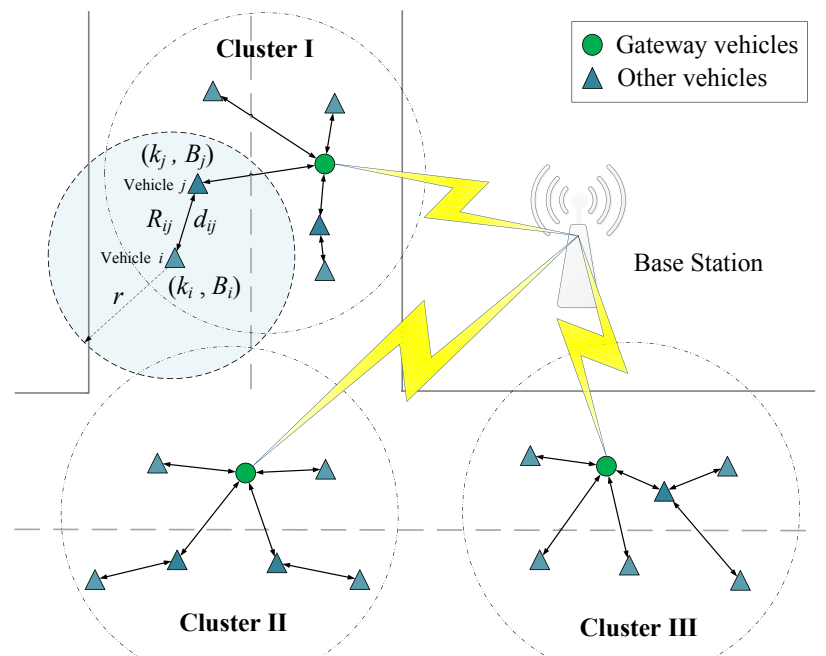

Fig. 2. The IoV aided traffic information collection architecture.

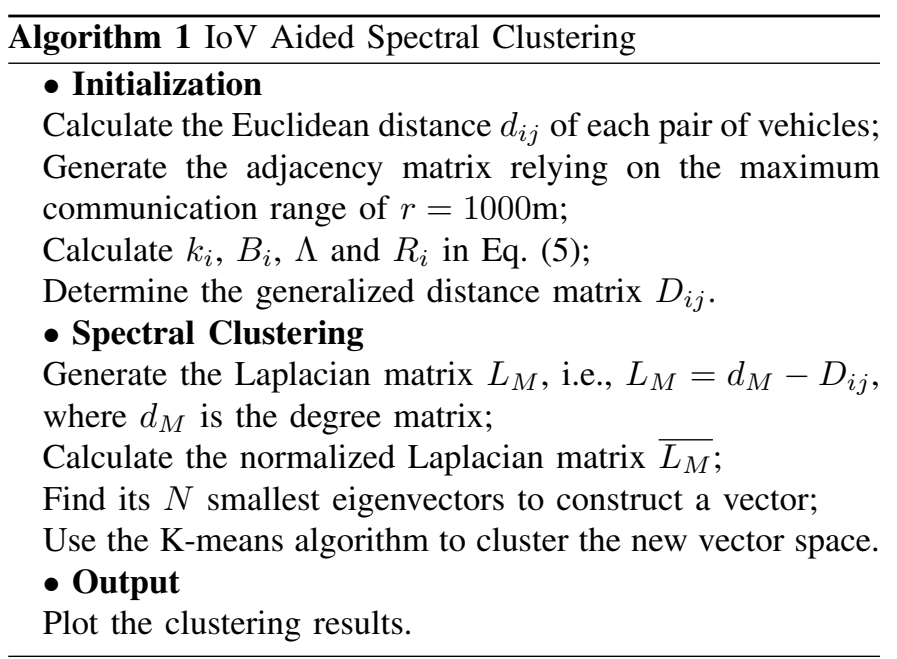

where $k_{i}$ represents the degree of node $i$ and $B_{i}$ denotes its betweenness centrality. Moreover, $\Lambda$ represents the throughput of a certain V2V or V2I link, as defined in Eq. (4). Furthermore, $\xi_{1}$ and $\xi_{2}$ represents the characteristic parameters, which depend on the network topology, while $\omega_{1}$ and $\omega_{2}$ are nonlinear control parameters invoked for the sake of flexibility.

Thus, relying on the concept of the node's communication impedance, we formulate the generalized distance $D_{i j}$ between vehicle $i$ and vehicle $j$ as:

$$
D_{i j}=\epsilon\left(R_{i}+R_{j}\right)+(1-\epsilon) d_{i j}
$$

where $R_{i}$ and $R_{j}$ represent the communication impedance of vehicle $i$ and vehicle $j$, respectively. Furthermore, $d_{i j}$ is the distance between the two vehicles, while $\epsilon$ denotes the weighting coefficient.

Based on the definition of the node's communication impedance, we propose a clustering-style subdomain partitioning algorithm, namely IoV aided spectral clustering, to conceive our IoV aided traffic information collection architecture of Algorithm 1. 


\section{B. Gateway Selection for Traffic Information Collection}

In each IoV subdomain, the vehicles collect the surrounding traffic information and transmit it to the gateway nodes for further processing. The location of the gateway nodes determines both the attainable communication efficiency and the associated overhead. In this subsection, the criterion of selecting the gateway nodes is that of maximizing the network capacity quantified in terms of the node's peak-load capacity, which can be formulated as:

$$
\Theta=\frac{M}{\max _{i}\left\{R_{i} q(i)\right\}},
$$

where we denote the delivery capacity of each vehicle by $M$, while $R_{i}$ is node $i$ 's communication impedance. Moreover, $q(i)$ represents the probability of any packet passing through node $i$.

In the IoV based vehicular network, the specific choice of the gateway, which is usually characterized by carrying a heavy tele-traffic load, has a substantial influence on the network's capacity. Therefore, it is of vital importance to study the optimal gateway selection strategy by formulating a network capacity optimization model.

In our model, Dijkstra's classic routing strategy is used for forwarding packets and each node has a first-in-first-out (FIFO) packet queue. Moreover, once a packet reaches its destination gateway, it is removed from the network.

Let $g_{s t}$ represent the number of the shortest paths emerging from the source node $s$ to the gateway $t$, and $n_{s t}^{i}$ denotes the number of the shortest paths via node $i$ from $s$ to $t$. Then we have:

$$
q(i)=\sum_{s(s \neq i)} \sum_{t(t \neq i)} p(s, t) \frac{n_{s t}^{i}}{g_{s t}}
$$

where $p(s, t)$ is the probability of a packet being selected for routing from the source node $s$ to the gateway $t$. If both the sources and gateway destinations are chosen uniformly for routing, $\Theta$ of Eq. (7) can be rewritten as:

$$
\Theta=\frac{M(N-1)(N-2)}{\max _{i}\left\{R_{i} \sum_{s(s \neq i)} \sum_{t(t \neq i)} \frac{n_{s t}^{i}}{g_{s t}}\right\}} .
$$

However, in our model, we specifically select the gateway node in order to maximize the network capacity $\Theta$ of Eq. (7) instead of using a uniform-selection strategy. The selection of the gateway is given by the probability $p(t)$, but we still assume that the source nodes are uniformly distributed and are independently selected. Hence, we have:

$$
p(s, t)=p(s) p(t)=\frac{p(t)}{N-1} .
$$

Then, the probability of any packet passing through node $i$ during routing can be calculated as follows:

$$
\begin{aligned}
q(i) & =\sum_{s(s \neq i)} \sum_{t(t \neq i)} p(s, t) \frac{n_{s t}^{i}}{g_{s t}} \\
& =\frac{1}{N-1} \sum_{s \neq i} \sum_{t \neq i} p(t) \frac{n_{s t}^{i}}{g_{s t}} .
\end{aligned}
$$

Moreover, we define $q(i \mid t)$ to represent the probability of a packet passing through node $i$ conditioned on its arrival at the gateway $t$, which is formulated as:

$$
q(i \mid t)=\frac{1}{N-1} \sum_{s(s \neq t, s \neq i)} \frac{n_{s t}^{i}}{g_{s t}} .
$$

Hence, $\Theta$ of Eq. (7) can be reformulated as:

$$
\begin{aligned}
\Theta & =\frac{M}{\max _{i}\left\{R_{i} q(i)\right\}} \\
& =\frac{M}{\max _{i}\left\{R_{i} \sum_{t} p(t) q(i \mid t)\right\}} .
\end{aligned}
$$

Therefore, the optimal gateway selection is formulated as the following optimization problem:

$$
\begin{aligned}
\max & \Theta \\
\text { s.t. } & 0 \leq p(t) \leq 1 \\
& \sum_{t} p(t)=1
\end{aligned}
$$

Hence, to maximize the capacity $\Theta$ of the vehicular network having $N$ nodes is equivalent to solving the following minmax problem:

$$
\begin{array}{ll}
\min & \max _{i}\left\{R_{i} \sum_{t} p(t) q(i \mid t)\right\} \\
\text { s.t. } & 0 \leq p(t) \leq 1, \\
& \sum_{t} p(t)=1 .
\end{array}
$$

After introducing the auxiliary variable of

$$
\Omega=\max _{i}\left\{R_{i} \sum_{t} p(t) q(i \mid t)\right\} \quad(i=1,2, \ldots, N),
$$

the optimization problem of Eq. (15) can be cast as the following linear programming problem:

$$
\begin{array}{cl}
\min & \Omega \\
\text { s.t. } & \mathbf{R A p}-\Omega \mathbf{1} \leq \mathbf{0}, \\
& \mathbf{p}^{T} \mathbf{1}=1, \\
& \mathbf{p} \geq \mathbf{0},
\end{array}
$$

where $\mathbf{A}=[q(i \mid t)], \mathbf{p}=[p(t), \quad t=1,2, \ldots, N]^{T}$ and $\mathbf{1}=$ $[1,1, \ldots, 1]$. Moreover, $\mathbf{R}$ is defined as:

$$
\mathbf{R}=\left[\begin{array}{llll}
R_{1} & 0 & \cdots & 0 \\
0 & R_{2} & & \vdots \\
\vdots & & \ddots & 0 \\
0 & \cdots & 0 & R_{N}
\end{array}\right] .
$$

Thus, we can readily find the minimum of $\Omega$ with the aid of linear programming algorithms. Furthermore, we can rewrite the linear programming problem of Eq. (17) relying on a slack variable $\mathbf{y}$, yielding

$$
\begin{aligned}
\min & \Omega \\
\text { s.t. } & \mathbf{R A} \mathbf{p}-\Omega \mathbf{1}+\mathbf{y}=\mathbf{0}, \\
& \mathbf{p}^{T} \mathbf{1}=1, \\
& \mathbf{y} \geq \mathbf{0}, \\
& \mathbf{p} \geq \mathbf{0} .
\end{aligned}
$$


Here, the new linear programming problem has $(2 N+1)$ variables, such as $\mathbf{p}, \mathbf{y}$ and $\Lambda$. Upon taking into account the constraints, we arrive at $(N+1)$ equalities, i.e., $\mathbf{p}^{T} \mathbf{1}=1$ as well as $\mathbf{R A p}-\Omega \mathbf{1}+\mathbf{y}=\mathbf{0}$. Therefore, based on simplex theory applied to our linear programming problem of Eq. (19), we can infer that at least $N$ of these $(2 N+1)$ variables are 0 . Given that $\Omega>0$, we have:

$$
N_{p(t)>0}=N-\chi(\mathbf{p}=\mathbf{0}) \leq \chi(\mathbf{y}=\mathbf{0}),
$$

where $\chi(\bullet=0)$ represents the number of 0 values in the vector "•".

Based on the above optimization framework, we can find our numerical solution relying on numerous iterative algorithms. The specific numerical solution algorithm we opted for is based on the obstacle function method [32], which is detailed in Section IV-C.

\section{IoV-Aided Information Flow Optimization}

Upon receiving any traffic control information, the gateways of each IoV subdomain broadcast the messages to the other vehicles. In order to support near-real-time traffic information broadcasting, a combination of multiple techniques will be considered.

The optimal user equilibrium (EU) is defined as the specific system state, in which any unilateral change degrades the objective function's $(\mathrm{OF})$ value in our communication system. Under the assumption that the impedances of all the links are known at a given time, we seek the optimal solution. For simplicity, we assume that there are certain packets that have to be transmitted from a source node to several gateways and vice versa. The total number of packets to be transmitted is denoted by $Q$. Moreover, $X=x_{1}, x_{2}, \ldots, x_{n}$ represents the total tele-traffic allocation set, where $x_{i}$ has to be routed through the $i$ th communication link. Again, Dijkstra's classic routing mechanism is considered [33], which finds the shortest path from a source vertex to a destination vertex, in a graph having weighted undirectional links. Hence, we define the OF $C(\mathbf{x})$ as:

$$
\begin{aligned}
C(\mathbf{x}) & =\sum_{i=1}^{n} C_{i}\left(x_{i}\right) \\
& =\sum_{i=1}^{n} \sum_{u, v} x_{i} R_{u v}^{i},
\end{aligned}
$$

where $R_{u v}^{i}$ is the communication impedance between node $u$ and $v$ along Dijkstra's path, when conveying the tele-traffic $x_{i}$. Let $c$ represent the maximum communication capacity of each communication link, which indicates the maximum number of communication tasks and $m_{u v}$ denotes the total communication tasks on the communication link between nodes $u$ and $v$, i.e., we have $m_{u v} \leq c$. Thus, we construct the following optimization problem:

$$
\begin{array}{ll}
\min & C(\mathbf{x})=\sum_{i=1}^{n} \sum_{u, v} x_{i} R_{u v}^{i} \\
\text { s.t. } & x_{i} \geq 0, \forall i=1,2, \ldots, n, \\
& \sum_{i=1}^{n} x_{i} \geq Q, \\
& m_{u v}=\sum_{i=1}^{n} x_{i} a_{u v}^{i} \leq c, \forall u, v \in V,
\end{array}
$$

where $\mathbf{x}=\left[x_{1}, x_{2}, \ldots, x_{n}\right]^{T}$. Specifically, we have $a_{u v}^{i}=1$, when the traffic $x_{i}$ is conveyed through the link connecting nodes $u$ and $v$, otherwise $a_{u v}^{i}=0$. The OF $C(\mathbf{x})$ has a linear form and the constraints are generalized inequalities. Then, the network's traffic allocation optimization problem can be deemed to be the convex optimization problem of Eq. (23),

$$
\begin{aligned}
\min & C(\mathbf{x}) \\
\text { s.t. } & \mathbf{x} \geq \mathbf{0}, \\
& \mathbf{x}^{T} \mathbf{1} \geq Q, \\
& \mathbf{A x} \leq c \mathbf{1},
\end{aligned}
$$

with the definition of the traffic-edge incidence matrix $\mathbf{A} \in$ $\mathbf{R}^{E \times n}$ given by:

$$
\mathbf{A}_{i j}= \begin{cases}1, & \text { traffic } j \text { passing the edge } i, \\ 0, & \text { otherwise, }\end{cases}
$$

where $E$ represents the total number of edges in the graph, $\mathbf{x}=\left[x_{1}, x_{2}, \ldots, x_{n}\right]^{T}$, and $\mathbf{1}=[1,1, \ldots, 1]^{T}$.

Due to the "small-world nature" of the links, the traffic-edge incidence matrix $\mathbf{A}$ is usually a sparse matrix. As a convex optimization problem, its standard form is given by:

$$
\begin{aligned}
\min & \mathbf{x}^{T} \mathbf{R}_{w} \\
\text { s.t. } & -\mathbf{x} \leq \mathbf{0}, \\
& Q-\mathbf{x}^{T} \mathbf{1} \leq 0, \\
& \mathbf{A x}-c \mathbf{1} \leq \mathbf{0},
\end{aligned}
$$

where $\mathbf{R}_{w}$ represents the sum of the communication impedances along each traffic allocation path. It is a vector optimization problem, which is subjected to generalized inequality constraints. Hence, a closed-form expression solution is difficult to obtain. Nevertheless, we incorporate an eigenfunction $I_{-}(u)$ to rewrite this linear programming problem, yielding:

$$
\begin{array}{ll}
\min & \mathbf{x}^{T} \mathbf{R}_{w}+\sum_{i=1}^{n+E+1} I_{-}\left[f_{i}(\mathbf{x})\right] \\
\text { s.t. } & f_{i}(\mathbf{x})=-x_{i}, i=1,2, \ldots, n, \\
& f_{i}(\mathbf{x})=Q-\mathbf{x}^{T} \mathbf{1}, i=n+1, \\
& f_{i}(\mathbf{x})=A_{i} \mathbf{x}-c, i=n+2, n+3, \ldots, n+E+1,
\end{array}
$$

where $I_{-}(u)=-(1 / t) \log (-u)$, and $A_{i}$ represents the row vector of the matrix $\mathbf{A}$, while the auxiliary variable of $t>0$ controls the computational accuracy. Then, we have the fol- 
lowing expression:

$$
\begin{array}{ll}
\min & \mathbf{x}^{T} \mathbf{R}_{w}+\sum_{i=1}^{n+E+1}-\frac{1}{t} \log \left[-f_{i}(\mathbf{x})\right] \\
\text { s.t. } & f_{i}(\mathbf{x})=-x_{i}, i=1,2, \ldots, n, \\
& f_{i}(\mathbf{x})=Q-\mathbf{x}^{T} \mathbf{1}, i=n+1, \\
& f_{i}(\mathbf{x})=A_{i} \mathbf{x}-c, i=n+2, n+3, \ldots, n+E+1 .
\end{array}
$$

Naturally, the logarithmic barrier function is defined as:

$$
\Phi(x)=-\sum_{i=1}^{m} \log \left[-f_{i}(x)\right]
$$

and the domain of $\Phi(x)$ is $\left\{x \in \mathbf{R}^{n} \mid f_{i}(\mathrm{x})<0, i=1, \ldots, m\right\}$. In [32], Boyd et al. have derived the gradient and Hessian matrix of the logarithmic barrier functions of:

$$
\nabla \Phi(x)=\sum_{i=1}^{m} \frac{1}{-f_{i}(x)} \nabla f_{i}(x),
$$

as well as

$$
\nabla^{2} \Phi(x)=\sum_{i=1}^{m} \frac{1}{f_{i}(x)} \nabla f_{i}(x) \nabla f_{i}(x)^{T}+\sum_{i=1}^{m} \frac{1}{-f_{i}(x)} \nabla^{2} f_{i}(x) .
$$

Considering the equivalence form of (27), we have:

$$
\begin{array}{ll}
\min & t \mathbf{x}^{T} \mathbf{R}_{w}+\sum_{i=1}^{n+E+1}-\log \left[-f_{i}(\mathbf{x})\right] \\
\text { s.t. } & f_{i}(\mathbf{x})=-x_{i}, i=1,2, \ldots, n, \\
& f_{i}(\mathbf{x})=Q-\mathbf{x}^{T} \mathbf{1}, i=n+1, \\
& f_{i}(\mathbf{x})=A_{i} \mathbf{x}-c, i=n+2, n+3, \ldots, n+E+1 .
\end{array}
$$

The solution of the optimization problem Eq. (31) is marked as $\mathbf{x}^{*}(t)$. Now we are in the position of proving that the deviation between $\mathbf{x}^{*}(t)$ and the optimal solution of the primal problem Eq. (25) is less than $(n+E+1) / t$. Therefore, we have to sequentially solve a series of convex optimization problems, and regard the present optimal solution as the initial point of the next-round of the optimization problem. Upon increasing $t$, the suboptimal solution is gradually approaching the primal problem's optimal solution. Furthermore, $\mathbf{x}^{*}(t)$ satisfies the Karush-Kuhn-Tucker condition [32], and we have:

$$
t \mathbf{R}_{w}-\frac{1}{\mathbf{x}}+\frac{1}{Q-\mathbf{x}^{T} \mathbf{1}} \cdot \mathbf{1}+\mathbf{A}^{T} \frac{1}{c \mathbf{1}-\mathbf{A x}}=\mathbf{0},
$$

where $\frac{1}{\mathbf{x}}=\left[\frac{1}{x_{1}}, \frac{1}{x_{2}}, \ldots, \frac{1}{x_{n}}\right]^{T}, \forall \mathbf{x} \in \mathbf{R}^{n}$. At the time of writing, it remains an open challenge to derive an analytical solution of the problem in Eq. (32). Hence, in Algorithm 2 we propose a solution relying on the classic Newton method of [34].

\section{Simulation Analysis}

In this section, we investigate the traffic information collection and diffusion over $\mathrm{IoV}$ networks relying on their topological time-invariance. Subsections V-A and V-B validate the time-invariant characteristics of the IoV network in terms of their parameters, such as the time-invariant clustering coefficient and the betweenness centrality. Subsection V-C
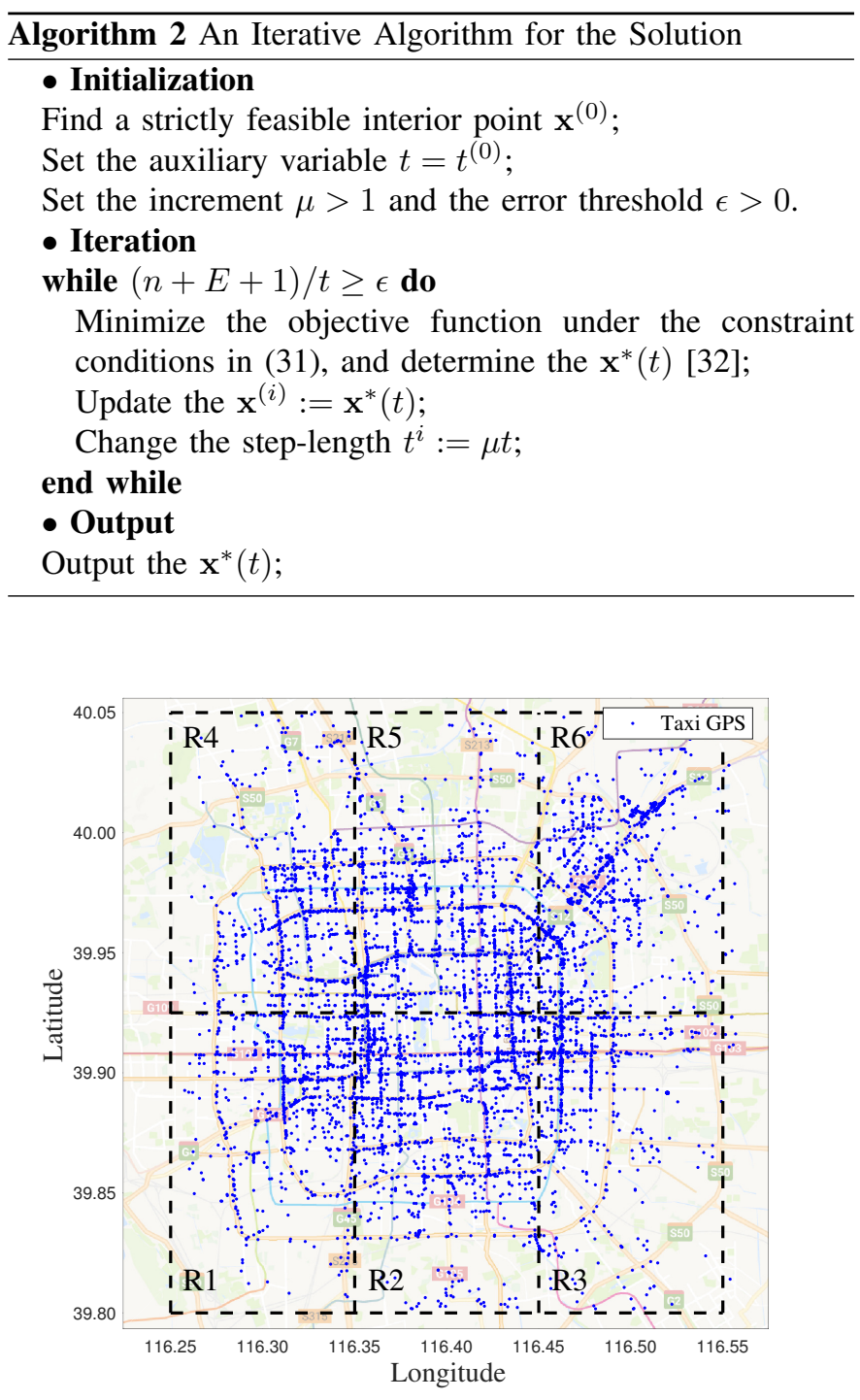

Fig. 3. Snapshot of the taxis' GPS coordinates in Beijing City (Longitude [116.25, 116.55], Latitude [39.80, 40.05]). Map layer from Google Maps.

characterizes the gateway selection performance based on our proposed algorithm.

\section{A. Data-Driven Complex IoV Networks}

We construct an IoV network relying on a real-world dataset, which contains the taxi GPS data of Beijing city (longitude from 116.25 to 116.55 , and latitude from 39.80 to 40.05) obtained from Microsoft Research Asia [27], for example. We first introduce the main parameters of the associated complex network and then discuss the statistical characteristics of this real-world data-set.

Based on the aforementioned taxi GPS dataset, Fig. 3 shows a snapshot of the taxis' position distribution at a certain time instant, which reflects the layout of Beijing city, including the roads and the partition of the downtown and suburban areas. According to the IEEE $802.11 \mathrm{p}$ standard [35], the maximum information transmission range is $r=1000 \mathrm{~m}$. Hence, we construct a distance-based vehicular network topology. In the 


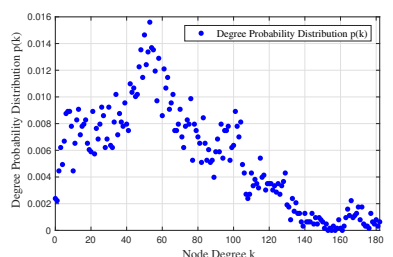

(a) Node Degree Distribution

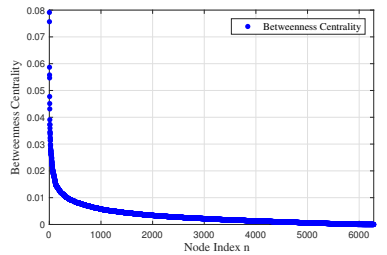

(c) Betweenness Centrality
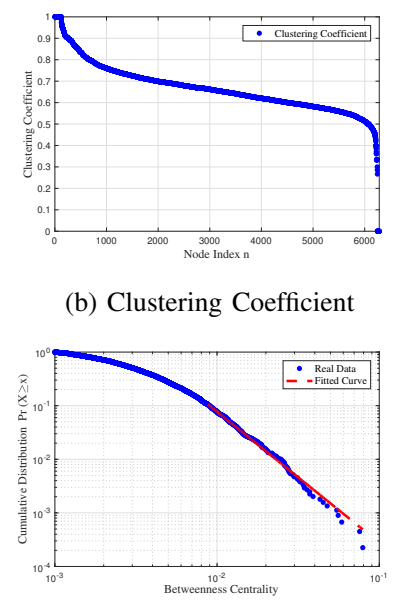

(d) Betweenness Centrality (Log-Log Coordinate) (b) Clustering Coefficient

Fig. 4. The complex network based parameters.

following, we verify the "small-world" property and "scalefree" nature of the IoV aided vehicular network in terms of the following topological parameters:

Node Degree Distribution: The node degree $k_{i}$ is defined as the number of other nodes the reference node is capable of communicating with. Moreover, $p(k)$ represents the probability that a random node's degree is $k$. Usually, the node distribution $p(k)$ of a real-world network obeys the Poisson distribution or the power-law distribution. A datadriven numerical simulation is conducted for our vehicular network (Longitude [116.25, 116.55], Latitude [39.8, 40.05]), for example. Fig. 4 (a) shows the node degree distribution of the network, which follows an approximate Poisson distribution.

Clustering Coefficients: Vehicle $i$ 's clustering coefficient is defined as:

$$
o_{i}=\frac{E_{i}}{k_{i}\left(k_{i}-1\right) / 2},
$$

where $k_{i}$ represents the node degree of vehicle $i$ and $E_{i}$ denotes the total number of the communication links among the neighbors of node $i$. This terminology indicates that the clustering coefficient characterizes the clustered versus dispersed nature of the network. Furthermore, the overall clustering coefficient of the entire network is the average of $o_{i}$. The dot symbols in Fig. 4 (b) characterize the clustering coefficients of all vehicles. Relying on the software Pajek ${ }^{3}$, the average network clustering coefficient can be calculated as $o=\frac{1}{N} \sum_{i=1}^{N} o_{i}=0.6666$.

Betweenness Centrality: Relying on the node degree, to a certain extent, we are capable of measuring the significance of each node. Specifically, the larger the node's degree, the more important role the node plays during the information transmission process. However, under some circumstances, a node having a low degree may also play a critical part by acting as a bridge when connecting two clusters. In order to accurately quantify the importance of node $i$, the normalized

\footnotetext{
${ }^{3}$ Pajek is an open source Windows program for analysis and visualization of large networks having some thousands or even millions of vertices.
}

betweenness centrality $B_{i}$ is defined as:

$$
B_{i}=\frac{2}{(N-1)(N-2)} \sum_{s \neq i \neq t} \frac{n_{s t}^{i}}{g_{s t}},
$$

where $g_{s t}$ represents the number of shortest paths leading from the source node $s$ to the destination node $t$, while $n_{s t}^{i}$ denotes the number of the shortest paths via node $i$ spanning from $s$ to $t$. Based on the definition in Eq. (34), we calculate the betweenness centrality of each node as indicated by Fig. 4 (c).

Average Path Length: The average path length $L$ represents the average number of hops in terms of the shortest multi-hop path. It measures the tightness of the vehicular network. Let $h_{i j}$ stand for the number of shortest path based hops between the communication link spanning from node $i$ to node $j$, i.e.,

$$
L=\frac{2}{N(N-1)} \sum_{i, j=1 ; i \geq j}^{N} h_{i j} .
$$

For each region in Fig. 3, we have $L_{1}=6.3623, L_{2}=6.5222$, $L_{3}=5.4683, L_{4}=6.4670, L_{5}=5.8115$, and $L_{6}=5.8657$.

According to the network clustering coefficient of $o=$ 0.6666 as well as to the network's average path length $\bar{L}=$ 6.0828 , the IoV based vehicular network is characterized by a high degree of clustering and a "six-degree" 4 average local path length, which conforms to the "small-world" property. Hence, the vehicular network can be deemed to be a local small-world complex network. Relying on dynamic theory and on synchronous control theory in the WS small-world model of [7] and in the NW small-world model of [36], we are now well placed for managing the information diffusion over vehicular networks. In Fig. 4 (d), the cumulative distribution function of the betweenness centrality is illustrated in a loglog coordinate form. The Kolmogorov-Smirnov (K-S) test's p-value benchmarked by the fitted power-law distribution is $p=0.19$. As for the scale-free nature of betweenness centrality based on Fig. 4 (d), we infer the conclusion that only a few nodes of our vehicular network play a critical role in the information diffusion process. It is beneficial for us to focus our attention on the vehicles at crossroad, as well as transportation hubs, acting as the bridging nodes.

\section{B. Time-Invariance Verification}

In the following, we analyze the taxis' GPS dataset at recorded at different times and verify the topological timeinvariance of our vehicular network.

Table I shows some of the network's topological parameters, such as the number of vehicles on the road, the average node degree, the node degree correlation ${ }^{5}$, the average shortest distance, the betweenness centrality and the clustering coefficient at different moments of the day. We can find that the number of vehicles and the average node degree varies with different times in a day. Specifically, given that 8:00 a.m. is the morning peak-time of Beijing city, numerous taxi drivers

\footnotetext{
${ }^{4}$ Six-degree separation is the idea that all living things and everything else in the world are six or fewer steps away from each other, i.e. two nodes can be connected via a maximum of six intermediate nodes in a large network.

${ }^{5}$ Degree correlation is for quantifying the preference of the connection of nodes.
} 
TABLE I

TOPOLOGICAL TIME-INVARIANCE VERIFICATION

\begin{tabular}{|c|c|c|c|c|c|c|}
\hline Time & No. of Vehicles ${ }^{1}$ & Av. Node Deg. ${ }^{2}$ & Deg. Corr. ${ }^{3}$ & Av. Dist. (m) ${ }^{4}$ & Betweenness & Clus. Coef. ${ }^{5}$ \\
\hline 8:00 a.m. & 4960 & 26.4091 & 0.9043 & 12844 & 0.0035 & 0.6749 \\
\hline 10:00 a.m. & 6870 & 34.8094 & 0.8290 & 11905 & 0.0027 & 0.6631 \\
\hline 12:00 noon & 7510 & 49.4798 & 0.8636 & 11628 & 0.0027 & 0.6666 \\
\hline 14:00 p.m. & 7475 & 48.3738 & 0.8485 & 11359 & 0.0028 & 0.6569 \\
\hline 16:00 p.m. & 7668 & 47.6251 & 0.8493 & 11305 & 0.0027 & 0.6583 \\
\hline 18:00 p.m. & 7863 & 47.6692 & 0.8413 & 11317 & 0.0026 & 0.6572 \\
\hline 20:00 p.m. & 7869 & 45.3365 & 0.8084 & 11472 & 0.0025 & 0.6616 \\
\hline
\end{tabular}

${ }^{1}$ Number of Vehicles.

2 Average Node Degree.

${ }^{3}$ Degree Correlation.

${ }^{4}$ Average Distance.

${ }^{5}$ Clustering Coefficient.

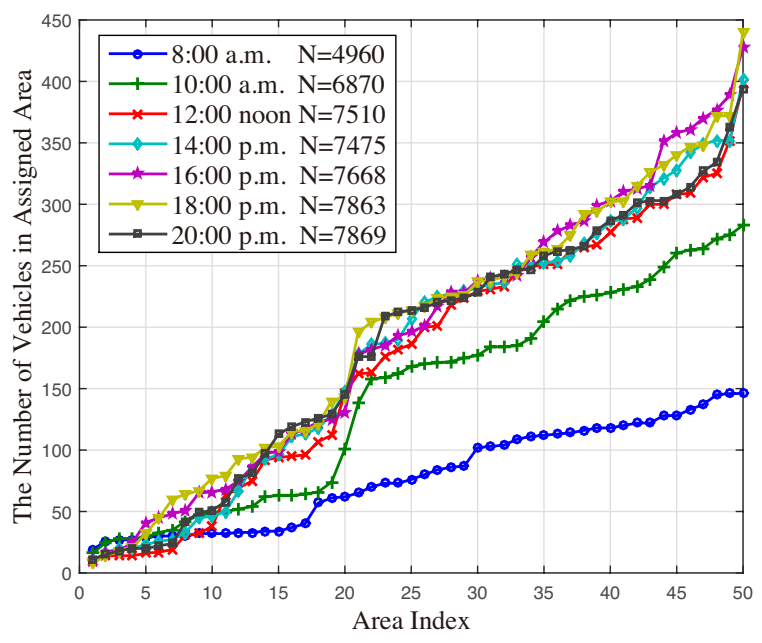

Fig. 5. The time-invariant spatial distribution.

may be unwilling to waste their time on the road because of the heavy traffic jam. Hence, the number of vehicles arrives its minimum. After 10:00 a.m., more and more taxies are on the road, which also results in a substantial increase of the average node degree. Although the node degree varies with time, the complex network based parameters remain steady. Based on considering the betweenness centrality and the clustering coefficient, we surmise that the vehicular network topology may be deemed time-invariant as well as exhibiting both the small-world property and scale-free property. Therefore, based on the above-mentioned complex network parameters, the topology of the IoV aided vehicular network may indeed be time-invariant from a macroscopic perspective. Naturally, the time-invariance is a statistical feature of the network topology, while the individual nodes have their own specific movement trajectory. Hence, we may consider a static topology at a specific moment for describing the associated dynamic graph. Both the time-invariant small-world property and scale-free property are beneficial in terms of managing the information routing, the optimization of the traffic allocation as well as in terms of designing the vehicular network structure.

The spatial distribution is a statistical feature, relying on

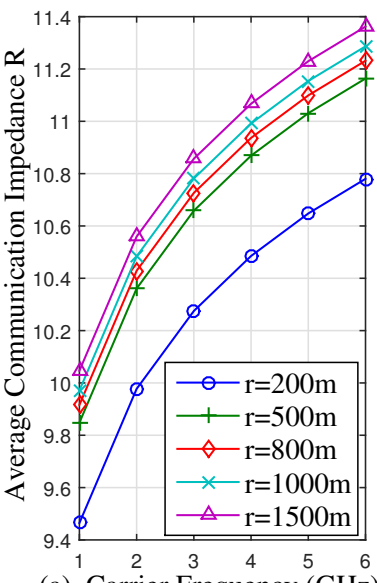

(a) Carrier Frequency $(\mathrm{GHz})$

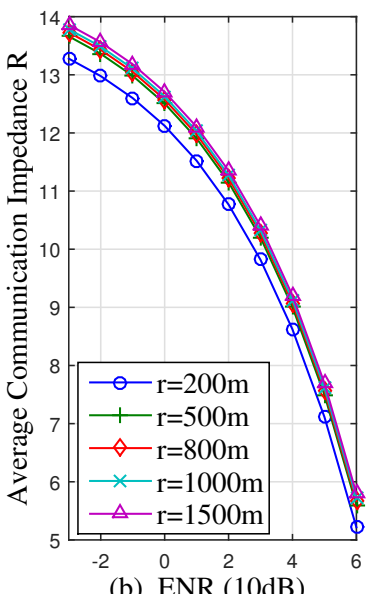

(b) ENR (10dB)
Fig. 6. The communication impedance versus the carrier frequency (a) and ENR (b) parameterized by the maximum coverage range.

the number of vehicles in specific areas. In our work, we randomly select 50 circular and non-overlapping regions with the radius of $1000 \mathrm{~m}$. Then, we calculate the total number of vehicles in each region at different moments. Fig. 5 shows the statistical results in ascending order. We can conclude that after 12:00 noon, the number of taxis on the road maintains a relatively stable value. Moreover, they follow a similar spatial distribution. Although the taxi-based vehicular network has fewer nodes in the morning, the normalized spatial distribution is similar to that recorded at other times, which is a manifestation of the spatial time-invariance. If we increase the number of sampling regions to 80 or more, similar conclusions will be valid.

\section{Traffic Information Collection and Diffusion}

Relying on the definition of the communication impedance, we analyzed the performance of traffic information collection and diffusion. In this subsection, we use the Matlab to evaluate our proposed algorithms. Concentrating on the topological properties of the vehicular network based on the taxis' GPS dataset in Beijing city, we provide constructive suggestions on the traffic management and the network design of ITS. In the 


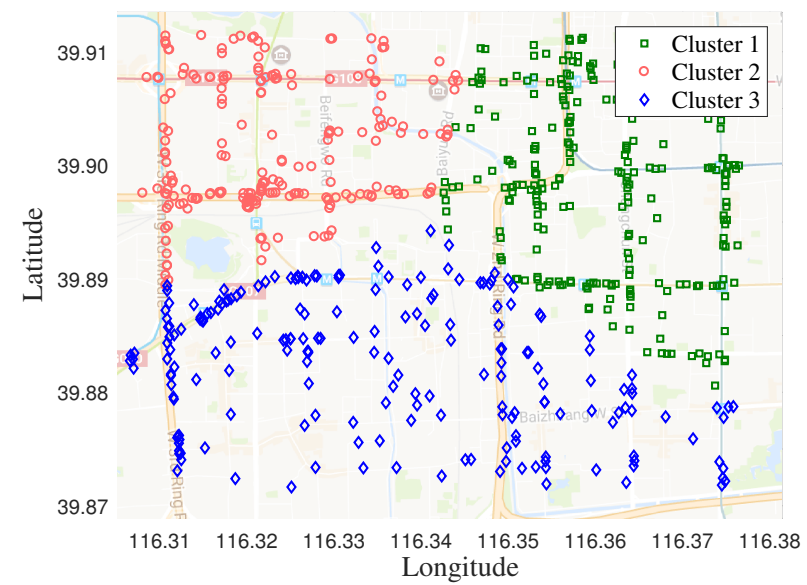

Fig. 7. Clustering results based on realistic distances (Tested on the subset with the longitude from 116.31 to 116.38 and the latitude from 39.87 to 39.91 relying on the maximum transmission range $r=1000 \mathrm{~m}$ ).

following, we study the factors influencing the communication impedance and information diffusion performance.

In our simulations, the wireless communication channel is modeled by a computationally efficient empirical obstacle model relying on $L_{x}=L_{\text {freespace }}+L_{\text {obs }}$, where $L_{\text {freespace }}$ and $L_{o b s}$ are defined in Eq. (1) and Eq. (2). Fig. 6 reflects the relationship between the communication impedance versus the carrier frequency as well as versus the ENR parameterized by the maximum communication range. We set the characteristic parameters of Eq. (3) to $\alpha_{1}=5 \times 10^{-6}, \alpha_{2}=2.5 \times 10^{-2}$, $\alpha_{3}=5$ and $\alpha_{4}=10^{-2}$. Moreover, the nonlinear control parameters of Eq. (3) are given by $\psi_{1}=1, \psi_{2}=0.8$ as well as $\psi_{3}=0.1$. In Fig. 6 (a), we compare the communication impedance versus the carrier frequency parameterized by the maximum communication range, where we assume $E N R=20 \mathrm{~dB}$. Upon increasing the carrier frequency, the average communication impedance $R$ of each scenario is also increased correspondingly. Likewise, a large maximum communication range $r$ contributes to increasing the average communication impedance owing to having an increased power loss. According to the IEEE 802.11p standard, in vehicular networks the maximum information transmission range is $r=1000 \mathrm{~m}$ and the carrier frequency is $f=5.9 \mathrm{GHz}$, which jointly determine the average communication impedance. To elaborate a little further, Fig. 6 (b) shows the relationship between the communication impedance and the $E N R$, where we adopt the standard carrier frequency of $f=5.9 \mathrm{GHz}$. All other parameters remain unchanged. Naturally, a high ENR leads to a low communication impedance. Since the ENR uniquely and unambiguously determines the received signal quality, the maximum information transmission range $r$ is expected to have no effect on the impedance.

Upon considering the subdomain segmentation of the traffic information collection architecture, Fig. 7 portrays our partitioning relying on realistic distances, where none of the remaining communication constraints are considered. Moreover, let $\xi_{1}=2.5, \omega_{1}=1, \epsilon=0.5$ and $\xi_{2} \Lambda^{\omega_{2}}$ be assumed to be constant. The performance of spectral clustering

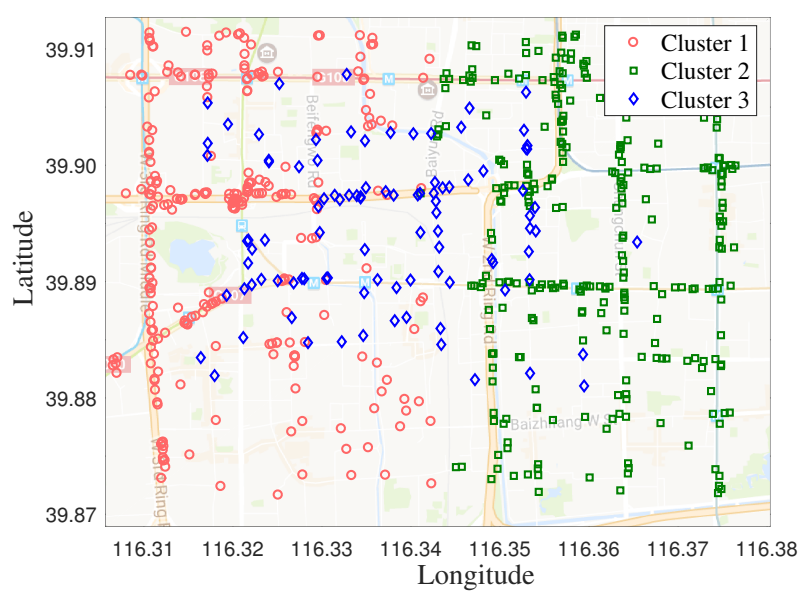

Fig. 8. Clustering results based on generalized distances (Tested on the same subset with the Fig. 7).

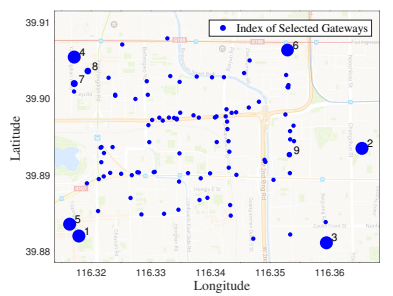

(a) Location of Selected Gateways.

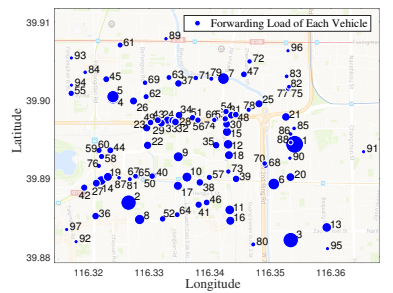

(c) Forwarding Load Distribution.

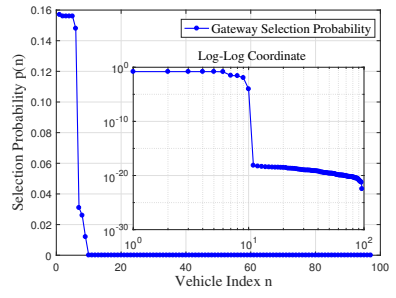

(b) Gateway Selection Probability.

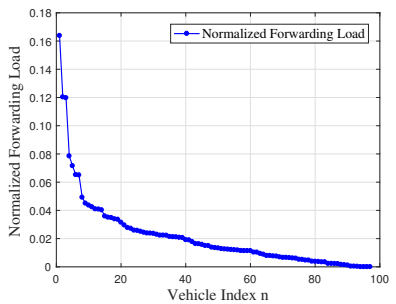

(d) Normalized Forwarding Load
Fig. 9. The simulation results of the optimal information gateway selection model defined in Eq. (14) (Tested on the subset with the longitude from 116.315 to 116.365 and the latitude from 39.88 to 39.91 relying on the maximum transmission range $r=1000 \mathrm{~m}$ ).

based on the generalized distances of Eq. (6) as described in Algorithm 1 is shown in Fig. 8. Relying on the clustering results, the average node's degree of Fig. 7 is 81.79 , while it is 71.30 for Fig. 8. Our proposed IoV aided architecture design may form irregular cluster shapes, but it has the minimum communication consumption considering the vehicle's location and load, which provides a constructive suggestion on the local traffic information collection.

As for the traffic information gateway selection model, Fig. 9 shows the related simulation results, where we also adopt the standard carrier frequency of $f=5.9 \mathrm{GHz}$ and maximum communication range of $r=1000 \mathrm{~m}$. Fig. 9 (a) portrays the location of each gateway node, where the size of the dot represents the probability of the node being selected as a gateway. In Fig. 9 (b), the selection distribution as well as its log-log coordinate form is shown. We can find that only 


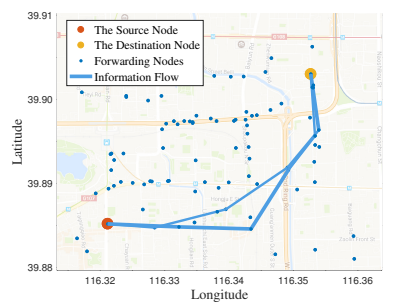

(a) Link's Capacity $c=640$

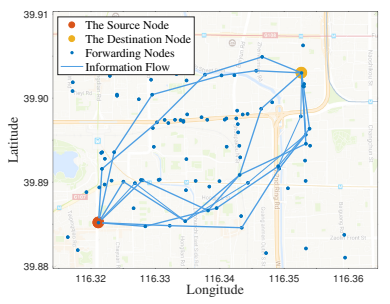

(c) Link's Capacity $c=160$

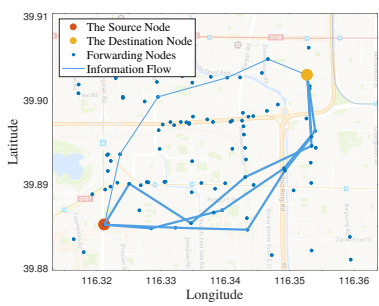

(b) Link's Capacity $c=320$

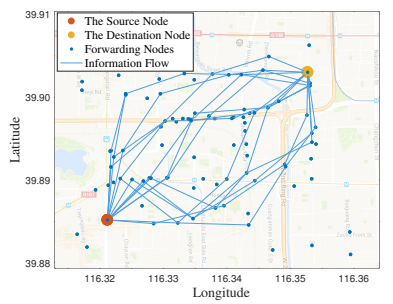

(d) Link's Capacity $c=80$
Fig. 10. The simulation results of information flow optimization model defined in Eq. (22 parameterized by different link's capacity $c$ of Eq. (21) (Tested on the subset with the longitude from 116.315 to 116.365 and the latitude from 39.88 to 39.91 relying on the maximum transmission range $r=1000 \mathrm{~m}$ and $Q=1000)$.

a few vehicles have a relatively high probability of acting as gateways in our heterogeneous IoV aided vehicular network. Fig. 9 (c) and (d) record the times of forwarding a packet of each node, namely the forwarding load, in the context of a broadcasting scenario from one gateway node selected according to our proposed gateway selection model to other vehicles. The size of the dots in Fig. 9 (c) represents the node's forwarding load. Moreover, we calculated their normalized forwarding load, which is shown in Fig. 9 (d). Specifically, in our paper, the gateway vehicle is responsible for gathering the information or for broadcasting the information gleaned from the other nodes to the control center, and vice versa. Explicitly, the gateways can be viewed as a bridge between the other vehicles and the command center. Moreover, our proposed gateway selection algorithm aims for maximizing the network capacity quantified by the node's peak-load capacity. As shown in Fig. 9 (c) and (d), vehicles in the vicinity of crossroads have a relatively high forwarding load. However, the internal nodes may be not capable of acting as gateway nodes. Therefore, the gateways are distributed near the border of the network, as shown in Fig. 9 (a).

As for our proposed traffic information flow optimization model of Section IV-C, Fig. 10 shows the planning path parameterized by the different links' capacity $c$ of Eq. (21) considering the communication impedance. The traffic information flow on each link is quantified by the width of the line in Fig. 10 and further characterized in Fig. 11.

\section{CONCLUSIONS}

In this paper, we studied the traffic information collection and diffusion issues of IoV networks. First of all, we analyzed the characteristics of the GPS dataset of Beijing city taxis and verified both the time-invariant small-world nature and the scale-free property of the IoV network. Secondly, we defined

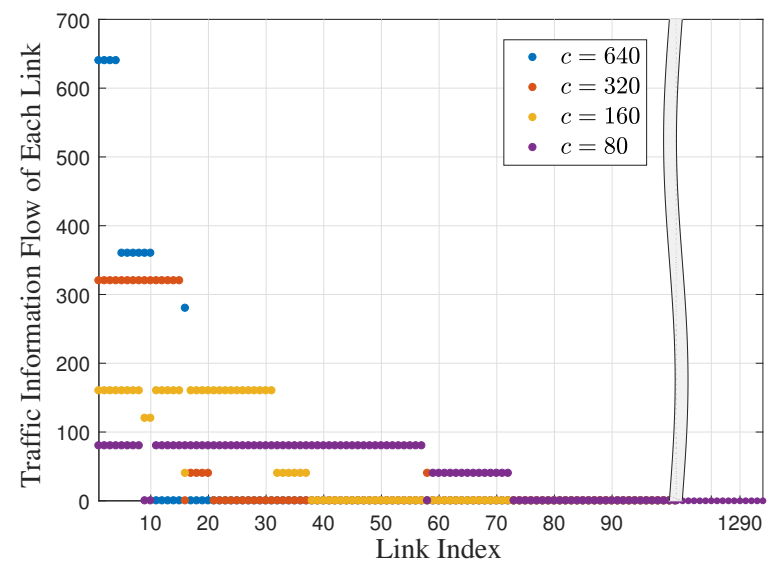

Fig. 11. Traffic information flow with respect to every link parameterized by different link's capacity $c$.

the link/node communication impedance and quantified the information collection and dissemination performance. Finally, we proposed an IoV aided local traffic collection architecture, a gateway selection scheme for information collection as well as an optimal traffic information transmission model for urban traffic control and management. Our simulation results show that only a few vehicles have a relatively high probability of acting as gateways and only certain routes should be selected as the information transmission path in our heterogeneous IoV aided vehicular network in order to achieve an improved transmission performance and reduced communication cost.

\section{REFERENCES}

[1] S. Grant-Muller and M. Usher, "Intelligent transport systems: The propensity for environmental and economic benefits," Technological Forecasting and Social Change, vol. 82, pp. 149-166, Feb. 2014.

[2] A. Festag, "Cooperative intelligent transport systems standards in Europe," IEEE Communications Magazine, vol. 52, no. 12, pp. 166-172, Dec. 2014.

[3] M. Gerla, E.-K. Lee, G. Pau, and U. Lee, "Internet of vehicles: From intelligent grid to autonomous cars and vehicular clouds," in 2014 IEEE World Forum on Internet of Things, Apr. 2014, pp. 241-246.

[4] N. Kumar, J. J. Rodrigues, and N. Chilamkurti, "Bayesian coalition game as-a-service for content distribution in internet of vehicles," IEEE Internet of Things Journal, vol. 1, no. 6, pp. 544-555, Dec. 2014.

[5] R. Yu, Y. Zhang, S. Gjessing, W. Xia, and K. Yang, "Toward cloudbased vehicular networks with efficient resource management," IEEE Network, vol. 27, no. 5, pp. 48-55, Otc. 2013.

[6] J. Dargay, D. Gately, and M. Sommer, "Vehicle ownership and income growth, worldwide: 1960-2030," The Energy Journal, vol. 28, no. 4, pp. 143-170, 2007.

[7] D. J. Watts and S. H. Strogatz, "Collective dynamics of small-world networks," Nature, vol. 393, no. 6684, pp. 440-442, Jun. 1998.

[8] A.-L. Barabási and R. Albert, "Emergence of scaling in random networks," Science, vol. 286, no. 5439, pp. 509-512, Oct. 1999.

[9] V. A. Butakov and P. A. Ioannou, "Personalized driver assistance for signalized intersections using V2I communication," IEEE Transactions on Intelligent Transportation Systems, vol. 17, no. 7, pp. 1910-1919, Jan. 2016.

[10] K. C. Dey, A. Rayamajhi, M. Chowdhury, P. Bhavsar, and J. Martin, "Vehicle-to-vehicle (V2V) and vehicle-to-infrastructure (V2I) communication in a heterogeneous wireless network-Performance evaluation," Transportation Research Part C: Emerging Technologies, vol. 68, pp. 168-184, Jul. 2016.

[11] C. E. Palazzi, M. Roccetti, and S. Ferretti, "An intervehicular communication architecture for safety and entertainment," IEEE Transactions on Intelligent Transportation Systems, vol. 11, no. 1, pp. 90-99, Mar. 2010 . 
[12] X. Cheng, L. Yang, and X. Shen, "D2D for intelligent transportation systems: A feasibility study," IEEE Transactions on Intelligent Transportation Systems, vol. 16, no. 4, pp. 1784-1793, Aug. 2015.

[13] L. Wischhof, A. Ebner, and H. Rohling, "Information dissemination in self-organizing intervehicle networks," IEEE Transactions on Intelligent Transportation Systems, vol. 6, no. 1, pp. 90-101, Mar. 2005.

[14] A. Agarwal, D. Starobinski, and T. D. Little, "Analytical model for message propagation in delay tolerant vehicular ad hoc networks," in Vehicular Technology Conference, 2008. VTC Spring 2008. IEEE. IEEE, May. 2008, pp. 3067-3071.

[15] M. Chaqfeh, A. Lakas, and I. Jawhar, "A survey on data dissemination in vehicular ad hoc networks," Vehicular Communications, vol. 1, no. 4, pp. 214-225, Sep. 2014.

[16] S. Panichpapiboon and W. Pattara-Atikom, "A review of information dissemination protocols for vehicular ad hoc networks," IEEE Communications Surveys \& Tutorials, vol. 14, no. 3, pp. 784-798, Aug. 2012.

[17] X. Zhu, Y. Li, D. Jin, and J. Lu, "Contact-aware optimal resource allocation for mobile data offloading in opportunistic vehicular networks," IEEE Transactions on Vehicular Technology, vol. 66, no. 8, pp. 73847399, Aug. 2017.

[18] Q. Zhang, H. Zheng, J. Lan, J. An, and H. Peng, "An autonomous information collection and dissemination model for large-scale urban road networks," IEEE Transactions on Intelligent Transportation Systems, vol. 17, no. 4, pp. 1085-1095, Apr. 2016.

[19] R. Kim, H. Lim, and B. Krishnamachari, "Prefetching-based data dissemination in vehicular cloud systems," IEEE Transactions on Vehicular Technology, vol. 65, no. 1, pp. 292-306, Jan. 2016.

[20] G. Rémy, S.-M. Senouci, F. Jan, and Y. Gourhant, "LTE4V2XłCollection, dissemination and multi-hop forwarding," in IEEE International Conference on Communications (ICC), Ottawa, Canada, Jun. 2012, pp. 120-125.

[21] _ - "LTE4V2X: LTE for a centralized VANET organization," in IEEE Global Telecommunications Conference (GLOBECOM), Kathmandu, Nepal, Dec. 2011, pp. 1-6.

[22] M. di Bernardo, A. Salvi, and S. Santini, "Distributed consensus strategy for platooning of vehicles in the presence of time-varying heterogeneous communication delays," IEEE Transactions on Intelligent Transportation Systems, vol. 16, no. 1, pp. 102-112, Feb. 2015

[23] I. Leontiadis, G. Marfia, D. Mack, G. Pau, C. Mascolo, and M. Gerla, "On the effectiveness of an opportunistic traffic management system for vehicular networks," IEEE Transactions on Intelligent Transportation Systems, vol. 12, no. 4, pp. 1537-1548, Dec. 2011.

[24] A. Skordylis and N. Trigoni, "Efficient data propagation in trafficmonitoring vehicular networks," IEEE Transactions on Intelligent Transportation Systems, vol. 12, no. 3, pp. 680-694, Sep. 2011.

[25] N. Cenerario, T. Delot, and S. Ilarri, "A content-based dissemination protocol for VANETs: Exploiting the encounter probability," IEEE Transactions on Intelligent Transportation Systems, vol. 12, no. 3, pp. 771-782, Sep. 2011.

[26] J. Wang, C. Jiang, L. Gao, S. Yu, Z. Han, and Y. Ren, "Complex network theoretical analysis on information dissemination over vehicular networks," in 2016 IEEE International Communication Conference, Kuala Lumpur, Malaysia, May 2016.

[27] J. Yuan, Y. Zheng, C. Zhang, W. Xie, X. Xie, G. Sun, and Y. Huang, "T-drive: driving directions based on taxi trajectories," in 18th SIGSPATIAL International Conference on Advances in Geographic Information Systems, New York City, NY, Nov. 2010, pp. 99-108.

[28] C. Sommer, D. Eckhoff, R. German, and F. Dressler, "A computationally inexpensive empirical model of IEEE $802.11 \mathrm{p}$ radio shadowing in urban environments," in 8th International Conference on Wireless On-Demand Network Systems and Services, Bardonecchia, Italy, Jan. 2011, pp. 8490.

[29] M. Barthelemy, "Betweenness centrality in large complex networks," The European Physical Journal B-Condensed Matter and Complex Systems, vol. 38, no. 2, pp. 163-168, Mar. 2004.

[30] A. El Gamal, M. Mohseni, and S. Zahedi, "Bounds on capacity and minimum energy-per-bit for AWGN relay channels," IEEE Transactions on Information Theory, vol. 52, no. 4, pp. 1545-1561, Apr. 2006.

[31] G.-M. Yang, C.-C. Ho, R. Zhang, and Y. Guan, "Throughput optimization for massive MIMO systems powered by wireless energy transfer,' IEEE Journal on Selected Areas in Communications, vol. 30, no. 60, pp. 1-12, Jan.

[32] S. Boyd and L. Vandenberghe, Convex optimization. Cambridge university press, 2004.

[33] S. Skiena, "Dijkstra's algorithm," Implementing Discrete Mathematics: Combinatorics and Graph Theory with Mathematica, Reading., pp. 225 227, 1990.
[34] R. W. Wedderburn, "Quasi-likelihood functions, generalized linear models, and the Gauss-Newton method," Biometrika, vol. 61, no. 3, pp. 439447, 1974.

[35] D. Jiang and L. Delgrossi, "IEEE 802.11p: towards an international standard for wireless access in vehicular environments," in IEEE 2008 Vehicular Technology Conference Spring, Singapore, May 2008, pp. 2036-2040.

[36] M. E. Newman and D. J. Watts, "Renormalization group analysis of the small-world network model," Physics Letters A, vol. 263, no. 4, pp. 341-346, Dec. 1999.

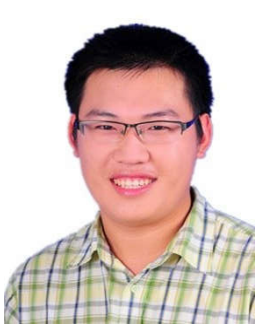

Jingjing Wang (S'14) received his B.S. degree in Electronic Information Engineering from Dalian University of Technology in 2014 with the highest honors. He currently works for his $\mathrm{PhD}$ degree at Complex Engineered Systems Lab (CESL) in Tsinghua University, Beijing. From Jan. 2016 to Mar. 2016, he visited Wireless Networks and Decision Systems (WNDS) Group, Singapore University of Technology and Design as a visiting student. Since Otc. 2017, he has been visiting the Telecommunications Group, University of Southampton as a joint $\mathrm{PhD}$ student. His research interests include the resource allocation an network association, learning theory aided modeling, analysis and signal processing, and information diffusion theory. He received Tsinghua GuangHua Scholarship in 2016 and graduate China National Scholarship Award in 2017.

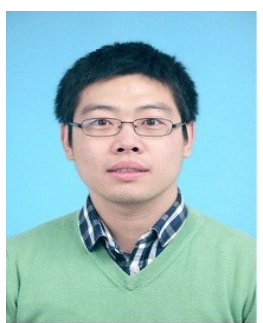

Chunxiao Jiang (S'09-M'13-SM'15) received the B.S. in information engineering from Beihang University in Jun. 2008 and the Ph.D. in electronic engineering from Tsinghua University in Jan. 2013, both with the highest honors. From Feb. 2013 - Jun. 2016, Dr. Jiang was a Postdoc in the Department of Electronic Engineering Tsinghua University, during which he visited University of Maryland College Park and University of Southampton. He is a recipient of the IEEE Globecom Best Paper Award in 2013, the IEEE GlobalSIP Best Student Paper Award in 2015, the IEEE IWCMC Best Paper Award in 2017, and the IEEE Communications Society Young Author Best Paper Award in 2017. Since 2015, Dr. Jiang became a IEEE Senior Member.

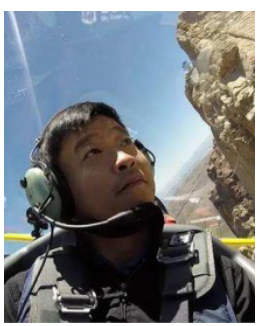

Zhu Han (S'01-M'04-SM'09-F'14) received the B.S. degree in electronic engineering from Tsinghua University, in 1997, and the M.S. and Ph.D. degrees in electrical and computer engineering from the University of Maryland, College Park, in 1999 and 2003, respectively. From 2000 to 2002, he was an R\&D Engineer of JDSU, Germantown, Maryland. From 2003 to 2006, he was a Research Associate at the University of Maryland. From 2006 to 2008, he was an assistant professor at Boise State University, Idaho. Currently, he is a Professor in the Electrical and Computer Engineering Department as well as in the Computer Science Department at the University of Houston, Texas. His research interests include wireless resource allocation and management, wireless communications and networking, game theory, big data analysis, security, and smart grid. Dr. Han received an NSF Career Award in 2010, the Fred W. Ellersick Prize of the IEEE Communication Society in 2011, the EURASIP Best Paper Award for the Journal on Advances in Signal Processing in 2015, IEEE Leonard G. Abraham Prize in the field of Communications Systems (best paper award in IEEE JSAC) in 2016, and several best paper awards in IEEE conferences. Currently, Dr. Han is an IEEE Communications Society Distinguished Lecturer. 


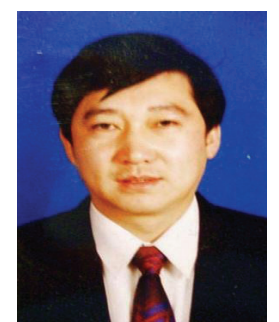

Yong Ren received his B.S, M.S and Ph.D. degrees in electronic engineering from Harbin Institute of Technology, China, in 1984, 1987, and 1994, respectively. He worked as a post doctor at Department of Electronics Engineering, Tsinghua University, China from 1995 to 1997 . Now he is a professor of Department of Electronics Engineering and the director of the Complexity Engineered Systems Lab in Tsinghua University. He holds 12 patents, and has authored or co-authored more than 100 technical papers in the behavior of computer network, P2P network and cognitive networks. He has serves as a reviewer of IEEE Transactions on Communications, Digital Signal Processing, Chinese Physics Letters, Chinese Journal of Electronics, Chinese Journal of Computer Science and Technology, Chinese Journal of Aeronautics and so on. His current research interests include complex systems theory and its applications to the optimization and information sharing of the Internet, Internet of Things and ubiquitous network, cognitive networks and Cyber-Physical Systems.

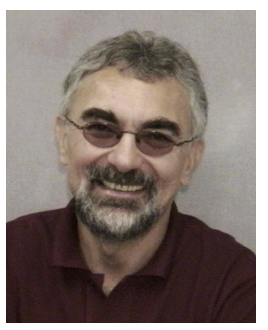

Lajos Hanzo (http://www-mobile.ecs.soton.ac.uk) FREng, FIEEE, FIET, Fellow of EURASIP, DSc received his degree in electronics in 1976 and his doctorate in 1983. In 2009 he was awarded an honorary doctorate by the Technical University of Budapest and in 2015 by the University of Edinburgh. In 2016 he was admitted to the Hungarian Academy of Science. During his 40-year career in telecommunications he has held various research and academic posts in Hungary, Germany and the UK. Since 1986 he has been with the School of Electronics and Computer Science, University of Southampton, UK, where he holds the chair in telecommunications. He has successfully supervised 111 $\mathrm{PhD}$ students, co-authored 18 John Wiley/IEEE Press books on mobile radio communications totalling in excess of 10000 pages, published 1703 research contributions at IEEE Xplore, acted both as TPC and General Chair of IEEE conferences, presented keynote lectures and has been awarded a number of distinctions. Currently he is directing a 60 -strong academic research team, working on a range of research projects in the field of wireless multimedia communications sponsored by industry, the Engineering and Physical Sciences Research Council (EPSRC) UK, the European Research Council's Advanced Fellow Grant and the Royal Society's Wolfson Research Merit Award. He is an enthusiastic supporter of industrial and academic liaison and he offers a range of industrial courses. He is also a Governor of the IEEE VTS. During 2008 - 2012 he was the Editor-in-Chief of the IEEE Press and a Chaired Professor also at Tsinghua University, Beijing. For further information on research in progress and associated publications please refer to http://wwwmobile.ecs.soton.ac.uk. Lajos has $30000+$ citations and an H-index of 72. 